\title{
Crescimento, Diarréia e Aleitamento Materno: 0 Caso da Vila do João ${ }^{1}$
}

\section{Growth, Diarrhea, and Breastfeeding: The Case of Vila do João}

\author{
Sonia A. Bittencourt ${ }^{2}$; Maria do Carmo Leal ${ }^{2}$ \\ Angela Maria Jourdan-Gadelha ${ }^{2} \&$ Maria Auxiliadora Oliveira ${ }^{3}$
}

\begin{abstract}
BITTENCOURT, S. A.; LEAL, M. C.; JOURDAN-GADELHA, A. M. \& OLIVEIRA, M. A. Growth, Diarrhea, and Breastfeeding: The Case of Vila do João. Cad. Saúde Públ., Rio de Janeiro, 9 (supplement 1): 07-13, 1993.
\end{abstract}

A prospective study on children under six months old living in an urban area on the periphery of Rio de Janeiro was carried out to examine the possible effect of diarrhea in differentiating the monthly growth rate in weight and height, according to type of nursing.

The results seem to indicate that infants show a delay in growth of weight and height as a result of greater incidence and prevalence of diarrhea.

It was also shown that human breast milk has a protective effect, reducing weight loss from episodes of diarrhea.

The study concludes that the promotion of breastfeeding, together with the control of diarrheal diseases, can provide immediate benefits in improving the nutritional status of the infant population.

Key words: Growth; Child Development; Diarrhea, Infantile; Breast Feeding

\section{INTRODUÇÃO}

Durante os primeiros 4-5 meses de vida, a velocidade de crescimento de crianças submetidas a condições de vida precárias é semelhante e, às vezes, superior àquela das diferentes populações de referência (Martorell \& Habicht, 1986; Rivera, 1988; Waterlow et al., 1980; Whitehead \& Paul, 1984). Entretanto, após este período, tal tendência se modifica: a taxa de

\footnotetext{
${ }^{1}$ Trabalho financiado pela Organização Pan-Americana da Saúde com o título "Características Epidemiológicas de Diarréias Infecciosas em uma Comunidade de Baixa Renda". Parte da análise foi desenvolvida no Instituto de Nutrición de Centroamérica y Panamá (INCAP), com bolsa de estudo outorgada pela Fundação Kellogg.

${ }^{2}$ Departamento de Epidemiologia e Métodos

Quantitativos em Saúde da Escola Nacional de Saúde

Pública. Rua Leopoldo de Bulhões 1480, Rio de Janeiro, RJ, 21041-210, Brasil.

${ }^{3}$ Departamento de Ciências Biológicas da Escola Nacional de Saúde Pública. Rua Leopoldo de Bulhões 1480, Rio de Janeiro, RJ, 21041-210, Brasil.
}

crescimento destas crianças diminui mais rapidamente que as das populações de referência (Waterlow et al., 1980).

Uma possível explicação para este padrão de crescimento seria que, até os 4-5 meses de vida, a quantidade e a qualidade do leite humano são adequadas para o crescimento normal, assim como para o crescimento compensatório após episódios de doença (Rowland et al., 1988).

As principais causas imediatas estudadas de falta de crescimento após o $4^{\circ}-5^{\circ}$ mês de vida são a inadequação alimentar; a ocorrência de doenças infecciosas, particularmente a doença diarréica (Martorell et al., 1975; Rowland et al., 1977); e a interação destes dois fatores (Habicht et al., 1974; Rivera et al., 1989).

Com o início do período de desmame, ocorre uma redução no consumo de alimentos, eleva-se a freqüência de doenças diarréicas e desaparece a proteção blindada pelo leite humano (Brown, 1978; Mata, 1983; Watkinson, 1981).

$\mathrm{O}$ efeito negativo das doenças diarréicas sobre o crescimento é exercido através de três meca- 
nismos interdependentes e interativos: a diminuição da quantidade de alimento ingerido, a má absorção de nutrientes e as mudanças metabólicas. Como conseqüência, ocorre perda aguda de peso e, nos casos de diarréia prolongada ou de episódios freqüentes de infecção, observa-se também a detenção do crescimento linear (Delgado et al., 1988; OPS, 1987).

Resultados anteriores demonstram que, nas crianças menores de 18 meses da Vila do João, a diarréia reduz, em média, 13,4 gramas de peso e $0,132 \mathrm{~mm}$ de comprimento por dia (Bittencourt et al., 1993).

Contudo, Hoyle (1980) observou que o aleitamento contínuo durante episódios agudos de diarréia protege a criança contra a redução do consumo de calorias e proteínas.

Este trabalho tem como objetivo verificar se, durante os primeiros 6 meses de vida, existe um efeito diferenciado da diarréia na velocidade de crescimento mensal em peso e em comprimento entre as crianças aleitadas e as totalmente desmamadas. $\mathrm{O}$ trabalho foi realizado numa área urbana da periferia da cidade do Rio de Janeiro. Esta constatação poderia indicar ações pertinentes e oportunas na área de saúde infantil.

\section{MATERIAL E MÉTODOS}

As informações contidas nos arquivos do banco de dados do estudo prospectivo "Características Epidemiológicas de Diarréias Infecciosas na Vila do João", realizado entre agosto de 1984 e setembro de 1985 , foram utilizadas para a presente análise de dados de antropometria, morbidade por diarréia e tipo de aleitamento materno das crianças menores de 6 meses, no período compreendido de janeiro a setembro de 1985.

A Vila do João é um bairro urbanizado de casas populares com sistema público de abastecimento de água, esgoto e luz elétrica. Existem alguns serviços públicos instalados, como a Unidade de Serviços Básicos de Saúde do Hospital da Universidade Federal do Rio de Janeiro, uma escola pública de $1^{\circ}$ grau, uma creche da Legião Brasileira de Assistência, uma unidade da Ação Comunitária do Brasil, um posto policial e algumas lojas comerciais.
$\mathrm{Na}$ época em que se realizou a investigação, a população local era de 8.000 habitantes, sendo que a maioria possuía baixo nível de escolaridade e renda familiar média mensal de US\$60.

A investigação consistiu no seguimento longitudinal inicial de uma coorte de crianças com idades entre 0 e 6 meses. Esta coorte foi acompanhada durante 1 ano, através de visitas semanais.

Como alguns agentes etiológicos da diarréia apresentam comportamento cíclico e estacional, decidiu-se obter, no semestre seguinte, uma nova coorte de crianças de 0 a 6 meses.

Para detalhes sobre a metodologia, consultar publicação anterior (Bittencourt et al., 1993).

\section{Análise Estatística}

A unidade de análise utilizada foi criançamês. Com o objetivo de caracterizar o crescimento das crianças estudadas, as medidas de peso e comprimento foram convertidas em velocidade de crescimento em peso e em comprimento entre duas medidas adjacentes e padronizadas em intervalo de 1 mês (dividindose a velocidade de crescimento pelo número de dias no intervalo e multiplicando-se o resultado pelo quociente $365 / 12$ ). Partindo do pressuposto de que a relação entre velocidade de crescimento em peso e em comprimento é linear nos 6 primeiros meses de vida, calculou-se o Percentil 50 (P50) da velocidade de peso e comprimento por mês, grupo de idade e sexo, comparando-o com o da população de referência do National Center of Health Statistics (Roche et al., 1989; Bittencourt et al., 1993).

A densidade de incidência (Kleinbaum et al., 1986a) de diarréia por grupo de idade foi calculada dividindo-se o número de episódios novos que ocorreram durante o intervalo entre duas medidas antropométricas adjacentes pelo número de dias de observação no mesmo período.

A prevalência de diarréia (Kleinbaum et al., 1986b) foi calculada dividindo-se o número de dias com diarréia durante o intervalo entre duas medidas adjacentes pelo número de dias de observação no período.

Para a descrição do aleitamento materno, utilizou-se o método de construção da Tabela de Vida, empregando-se o estimador KaplanMeier (Kaplan \& Meier, 1958). 
Com o objetivo de verificar se a doença diarréica e o tipo de aleitamento apresentam efeitos interativos sobre o crescimento de crianças menores de 6 meses, ou seja, se o efeito negativo da diarréia no crescimento é maior nas crianças totalmente desmamadas, utilizou-se um modelo de regressão múltipla. Ao se representar graficamente a relação entre velocidade de crescimento em peso e idade e de comprimento e idade das crianças menores de 6 meses, observou-se uma relação linear entre as variáveis. Por conseguinte, o modelo proposto foi:

$$
\begin{aligned}
& Y_{1}=\beta_{0}+\beta_{1} x_{1}+\beta_{2} x_{2}+\beta_{3} x_{3}+\beta_{4} x_{1} \cdot x_{2} \\
& Y_{2}=\beta_{0}+\beta_{1} x_{1}+\beta_{2} x_{2}+\beta_{3} x_{3}+\beta_{4} x_{1} \cdot x_{2}
\end{aligned}
$$

Neste modelo $Y_{1}$ é a velocidade de peso mensal e $\mathrm{Y}_{2}$ é a velocidade de comprimento mensal. Ademais, $x_{1}$ é a prevalência de diarréia; $x_{2}$, o tipo de aleitamento materno (codificado, na análise, como (1) para o desmame total e (0) para os aleitamentos exclusivo e parcial); e $\mathrm{x}_{3}$, a idade ao final do intervalo.

$O$ modelo incluiu a interação entre tipo de aleitamento e prevalência de diarréia $\left(\mathrm{x}_{1} \cdot \mathrm{x}_{2}\right)$.

$\mathrm{O}$ uso de um modelo de regressão múltipla que utiliza criança-mês como unidade de análise é imperfeito, pois ele não leva em consideração o efeito de conglomerado na estimativa do desvio padrão. Isto significa que as observações em uma mesma criança provavelmente estão correlacionadas. Caso não se considere o efeito de conglomerado, o desvio padrão pode estar errado.

Entretanto, ao se utilizar um fator de correção para que o efeito de conglomerado seja levado em consideração no modelo, comprova-se que os graus de significação estatística alcançados com e sem o fator de correção são muito similares (Rivera \& Martorell, 1988). Desta forma, os resultados obtidos com o modelo de regressão múltipla são apresentados utilizando-se a unidade de análise criança-mês.

\section{RESULTADOS E DISCUSSÃO}

Entre janeiro e setembro de 1985, foram acompanhadas 91 crianças de 0 a 6 meses de idade. Durante este período, ocorreram sete perdas $(7,7 \%)$.
O P50 da velocidade de peso nos 3 primeiros meses de vida das crianças estudadas foi de 873 g/mês e de $793 \mathrm{~g} / \mathrm{mês}$ para meninas e meninos, respectivamente. O P50 da velocidade de peso diminuiu marcadamente entre 4 e 6 meses de idade, sendo de $434 \mathrm{~g} / \mathrm{mês}$ para as meninas e de $456 \mathrm{~g} / \mathrm{mês}$ para os meninos, conforme observado nos Gráficos 1 e 2, respectivamente.

No Gráfico 1, observa-se que os meninos apresentaram o P50 da velocidade de peso abaixo do P10 do NCHS desde o nascimento. As meninas mostraram um melhor desempenho: o P50 da velocidade de peso nos 3 primeiros meses de vida situou-se entre o P25 e o P50 da população de referência, mas a partir do $4^{\circ}$ mês de vida observa-se um descenso, ficando o mesmo localizado entre o P25 e o P10 desta população.

FIGURA 1. Velocidade Mensal de Crescimento em Peso de Meninos por Grupos de Idade, em Relação ao NCHS, Vila do João, 1985

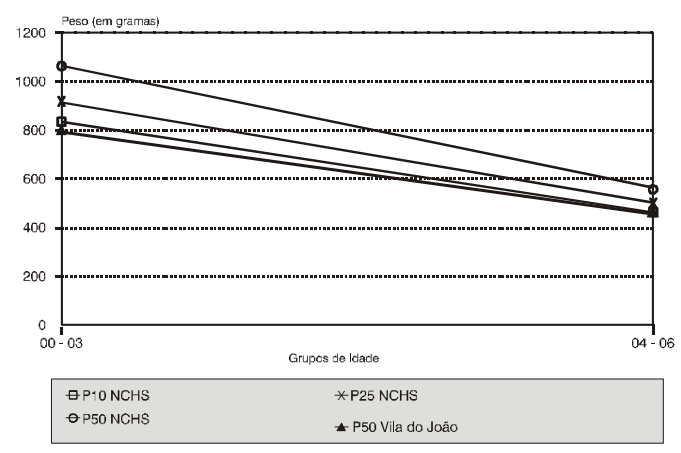

FIG URA 2. Velocidade Mensal de Crescimento em Peso de Meninas por Grupos de Idade, em Relação ao NCHS, Vila do João, 1985

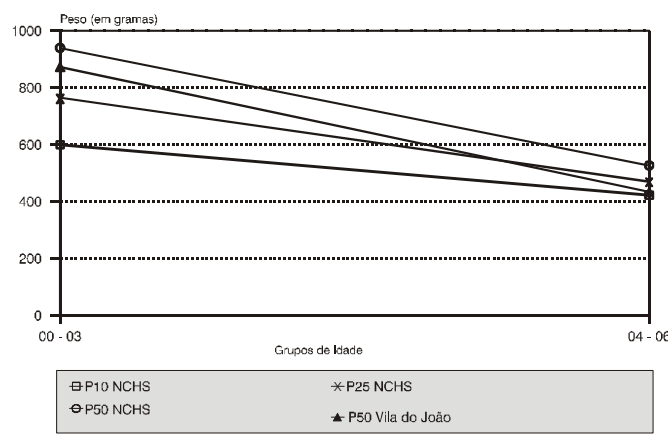


Em relação ao P50 da velocidade de comprimento das crianças estudadas, foram encontrados $2,92 \mathrm{~cm} /$ mês para as meninas e $3,13 \mathrm{~cm} / \mathrm{mês}$ para os meninos nos 3 primeiros meses de vida. De 4 a 6 meses de vida ocorreu uma acentuada desaceleração da velocidade de crescimento, sendo de $1,60 \mathrm{~cm} / \mathrm{mês}$ para ambos os sexos, como pode-se ver nos Gráficos 3 e 4 .

O P50 da velocidade de comprimento situouse entre o P50 e o P25 do NCHS para ambos os sexos até o $3^{\circ}$ mês de vida; a partir do $4^{\circ}$ mês de vida, este caiu para abaixo do P5.

As crianças estudadas apresentaram déficit de crescimento, o qual se agravou com o aumento

FIGURA 3. Velocidade Mensal de Crescimento em Meninos por Grupos de Idade, em Relação ao NCHS, Vila do João, 1985

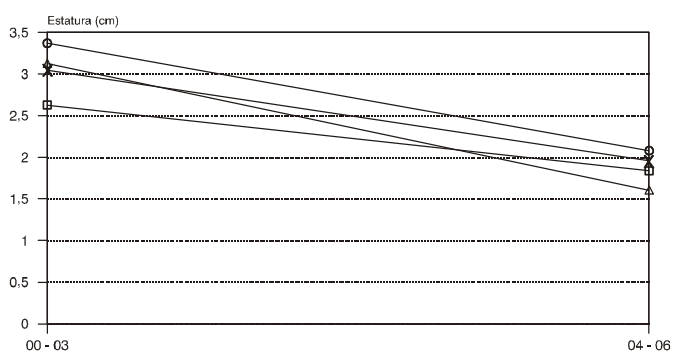

Grupos de Idade AP10 NCHS *P25 NCHS $\ominus$ P50 Vila do João $₫ P 50$ NCHS
FIGURA 4. Velocidade Mensal de Crescimento em Meninas por Grupos de Idade, em Relação ao NCHS, Vila do João, 1985

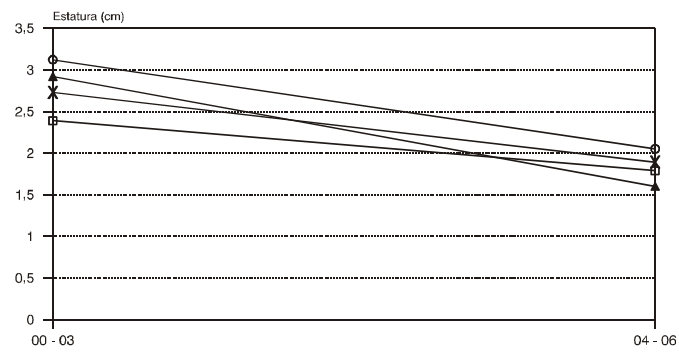

Grupos de ldade
૯P10 NCHS AP25 NCHS $\theta$ P50 Vila do Joáo ^P50 NCHS

da idade, sendo o crescimento linear o mais afetado. Os possíveis fatores agudos que poderiam estar interferindo no crescimento das crianças estudadas são uma alimentação inadequada, a ocorrência de doenças infecciosas (principalmente a diarréia) e a interação destes dois fatores.

Quanto ao padrão de aleitamento materno das crianças estudadas, como podemos visualizar na Tabela 1, o desmame precoce foi violento. Já na $1^{\text {a }}$ semana de vida ocorreu o desmame parcial em 18,44\% das crianças, e aos 30 dias somente $48,04 \%$ ainda estavam sendo aleitadas exclusivamente.

TABELA 1. Proporção Acumulada Estimada da Duração do Aleitamento Exclusivo e do Desmame Total por Idade ${ }^{(1)}$, Vila do João, 1985

\begin{tabular}{|c|c|c|}
\hline \multirow[b]{2}{*}{ Idade } & Tipo de Aleitamento & \multirow[b]{2}{*}{$\begin{array}{c}\text { Desmame Total } \\
\% \text { Acumulado }\end{array}$} \\
\hline & $\begin{array}{c}\text { Aleitamento Exclusivo } \\
\% \text { Acumulado }\end{array}$ & \\
\hline $1 \mathrm{dia}$ & 98,88 & - \\
\hline Até 6 dias & 81,56 & 3,85 \\
\hline Até 15 dias & 66,48 & 7,16 \\
\hline Até 30 dias & 48,04 & 11,03 \\
\hline Até 60 dias & 32,30 & 19,36 \\
\hline Até 90 dias & 19,84 & 28,41 \\
\hline Até 120 dias & 13,44 & 39,93 \\
\hline Até 150 dias & 9,25 & 44,66 \\
\hline Até 180 dias & 3,99 & 47,25 \\
\hline Até 215 dias & 0,66 & 50,87 \\
\hline
\end{tabular}

(1) Método Kaplan-Meier 
Verificou-se que o tempo médio estimado de aleitamento exclusivo foi de 55,9 dias, sendo a mediana estimada em 30 dias. O $1^{\circ}$ quartil foi estimado em 90 dias e o $3^{\circ}$ quartil, em 10 dias, o que indica que, com 10 dias de vida, $25 \%$ das crianças já se encontravam parcialmente desmamadas.

$\mathrm{O}$ aleitamento materno exclusivo por pelo menos 4 a 6 meses, proposto por programas de incentivo ao aleitamento materno, está longe de ser alcançado na Vila do João. Com 3 meses de vida, apenas $19,84 \%$ das crianças ainda estavam sendo aleitadas exclusivamente.

Também verificou-se uma duração do aleitamento materno total reduzida. Aos 3 meses de vida, cerca de $40 \%$ das crianças já se encontravam totalmente desmamadas. A duração média estimada do aleitamento total foi de 290,67 dias, sendo a mediana estimada em 209 dias.

Esta situação, provavelmente aliada a um acesso limitado à alimentação a partir do período de desmame, impossibilita o crescimento satisfatório das crianças da Vila do João, o qual pode ser agravado por episódios de infecção, que, além de aumentarem as necessidades nutricionais, reduzem a ingestão e a absorção de nutrientes.

Como mostra a Tabela 2, a densidade de incidência de diarréia foi de 4,01 por criança-ano e a prevalência de diarréia foi de $6,43 \%$.

Observa-se uma tendência de elevação dos indicadores de morbidade com o aumento da idade. Comparando-se estes resultados com os de uma área urbana do Nordeste brasileiro (Guerrant et al., 1983) com acesso a sistema de abastecimento de água e de esgoto, cuja morbidade por diarréia foi similar à observada em países desenvolvidos, os valores encontrados na Vila do João são elevados. A transmissão de organismos patógenos possivelmente se mantém, dentre outros fatores, devido a uma higiene pessoal, doméstica e de alimentos deficiente, além da alta densidade domiciliar.

TABELA 2. Densidade de Incidência e Prevalência de Diarréia segundo Grupo de Idade, Vila do João, 1985

\begin{tabular}{lcc}
\hline \hline $\begin{array}{l}\text { Grupo } \\
\text { de }\end{array}$ & $\begin{array}{c}\text { Densidade } \\
\text { de } \\
\text { Incidência } \\
\text { (meses) }\end{array}$ & $\begin{array}{c}\text { Prevalência } \\
\text { de } \\
\text { Diarréia } \\
\%\end{array}$ \\
\hline $0-3$ & 3,33 & 5,51 \\
$4-6$ & 4,37 & 6,85 \\
\hline Total & 4,01 & 6,43 \\
\hline \hline
\end{tabular}

A Tabela 3 apresenta o resultado do modelo de regressão no qual analisa-se o efeito da diarréia na velocidade de peso e comprimento por mês de crianças de 0 a 6 meses aleitadas exclusiva e parcialmente, em comparação com crianças desmamadas totalmente.

TA BELA 3. Efeito do Tipo de Aleitamento Materno e Prevalência de Diarréia na Velocidade Mensal de Peso e Comprimento de Crianças de 0 a 6 Meses, Vila do João

\begin{tabular}{|c|c|c|c|c|c|c|}
\hline \multirow{3}{*}{$\begin{array}{c}\text { Variável Dependente } \\
\text { n }\end{array}$} & \multirow{2}{*}{\multicolumn{3}{|c|}{$\frac{\text { Peso }(\mathrm{g})^{(2)}}{212}$}} & \multicolumn{3}{|c|}{ Comprimento (mm) ${ }^{(3)}$} \\
\hline & & & & & 123 & \\
\hline & $\begin{array}{c}\text { Coef. de } \\
\text { Regressão }\end{array}$ & $\begin{array}{c}\text { Erro } \\
\text { Padrão }\end{array}$ & $\mathrm{P}$ & $\begin{array}{c}\text { Coef. de } \\
\text { Regressão }\end{array}$ & $\begin{array}{c}\text { Erro } \\
\text { Padrão }\end{array}$ & $\mathrm{P}$ \\
\hline Intercepto & 1208,41 & 70,27 & 0,0001 & 42,78 & 2,46 & 0,0001 \\
\hline Prevalência de Diarréia (D) & $-4,08$ & 2,15 & 0,0589 & $-0,05$ & 0,07 & 0,4475 \\
\hline Idade & $-128,23$ & 15,04 & 0,0001 & $-4,28$ & 0,53 & 0,0001 \\
\hline Tipo de Aleitamento (A) ${ }^{(1)}$ & 85,73 & 55,25 & 0,1223 & $-0,98$ & 2,27 & 0,6665 \\
\hline Interação (AxD) & $-7,85$ & 3,11 & 0,0124 & $-0,05$ & 0,11 & 0,6533 \\
\hline
\end{tabular}

(1) Desmame Total $=1$

Aleitamento Exclusivo e Parcial $=0$

$\mathrm{R}^{2}$ Ajustado $=0,3363(2)$

$0,3846 \quad(3)$ 
O coeficiente do termo de interação foi significativo somente para a velocidade mensal de peso, sugerindo que o efeito negativo da diarréia é maior nas crianças desmamadas, mas só a partir de uma determinada prevalência de diarréia.

A falta de significância no termo de interação para o comprimento pode ser explicada pelo o fato de o déficit dessa medida ser acumulativo. Provavelmente, aumentando o intervalo entre as medições, o efeito poderá ser biologicamente importante.

A partir do modelo anterior, foram obtidas as linhas de regressão da prevalência de diarréia sobre a velocidade mensal de peso para as crianças aleitadas e para as desmamadas totalmente, utilizando como exemplo uma criança de 4 meses (média de idade dos menores de 6 meses), como mostra o Gráfico 5.

FIGURA 5. Linhas de Regressão da Velocidade Mensal de Peso segundo Tipo de Aleitamento e Prevalência de Diarréia, Vila do João, 1985

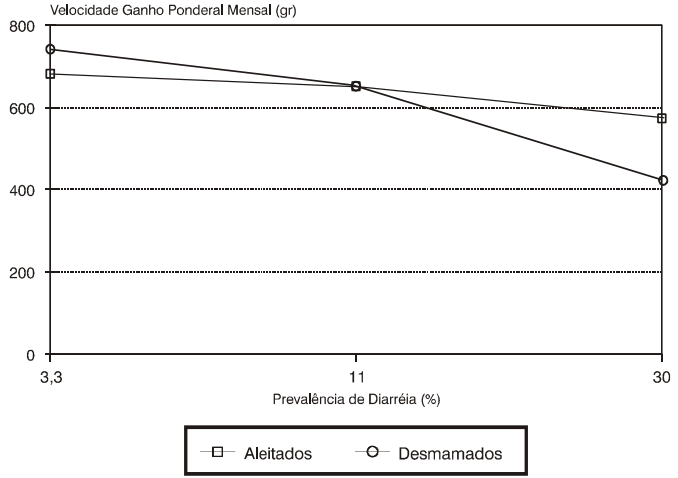

Estes resultados sugerem que o fato de a criança estar sendo amamentada é efetivo no sentido de atenuar o efeito adverso da diarréia na velocidade mensal de peso. Rowland et al. (1988) encontraram resultados semelhantes, os quais revelam haver uma dramática diferença do efeito da diarréia na velocidade de crescimento mensal de peso nos desmamados totalmente e nas crianças aleitadas exclusivamente.

\section{CONCLUSÃO}

Com os resultados encontrados, pode-se concluir que é necessário que haja uma estrutu- ração sistemática, contínua e dinâmica da atividade de promoção do aleitamento materno, com o objetivo de prolongar a sua prática. Tal necessidade justifica-se não só pelas propriedades antiinfecciosas do aleitamento materno, mas também pelo seu efeito protetor, reduzindo a perda de peso em função dos episódios diarréicos e proporcionando, assim, benefícios imediatos à população infantil.

\section{AGRADECIMENTO}

As autoras agradecem a Silvana Granado e Cirlene Rasina por suas contribuições ao trabalho de campo.

\section{RESUMO}

BITTENCOURT, S. A.; LEAL, M. C.; JOURDAN-GADELHA, A. M. \& OLIVEIRA, M. A. Crescimento, Diarréia e Aleitamento Materno: O Caso da Vila do João. Cad. Saúde Públ., Rio de Janeiro, 9 (suplemento 1): 07-13, 1993.

Mediante um estudo de coorte prospectivo em crianças menores de 6 meses residentes numa área urbana da periferia da cidade do Rio de Janeiro, estudou-se o possível efeito da diarréia na diferenciação da velocidade mensal de peso e comprimento, segundo tipo de aleitamento materno.

Os resultados parecem evidenciar que as crianças apresentam atrasos no crescimento pondo-estatural em razão da maior incidência e maior prevalência de diarréia.

Demonstrou-se, também, que o leite humano apresenta um efeito protetor, reduzindo a perda de peso em função dos episódios diarréicos.

Conclui-se que a promoção do aleitamento materno, aliada ao controle das doenças diarréicas, pode proporcionar benefícios imediatos na melhoria do estado nutricional da população infantil.

Palavras-Chave: Crescimento; Desenvolvimento Infantil; Diarréia Infantil; Aleitamento Materno 


\section{REFERÊNCIAS BIBLIOGRÁFICAS}

BROWN, K. H., 1978. Weaning in developing countries. A merican Journal of Clinical Nutrition, 31: 2066-2072.

BITTENCOURT, S. A.; LEAL, M. C. \& RIVERA, J., 1993. Diarrea y crecimiento en niños menores de 18 meses en Rio de Janeiro. Boletín de la Oficina Sanitaria Panamericana, 114: 193-201.

DELGADO, H.; GARCIA, B. \& HURTADO, E., 1988. Crecimiento físico, nutrición en los primeros años de vida. In: Crecimiento y desarrollo. Hechos y tendencias (M. Cuminsky; E. Moreno \& E. Ojeda, eds.), pp. 250-276, Washington, DC: OPS. (Publicación Científica, 510)

GUERRANT, R. L.; KIRCHOOFF, L.; SHIELDS, D. S.; NATIONS, M. K.; LESLIE, J.; SOUSA, M. A.; ARAÚJO, J. G.; CORREIA, L. L.; SAUER, K. T.; McCLELLANG, K. E.; TROWGRIDE, F. L. \& HUGHERS, J. M., 1983. Prospective study of diarrheal illnesses in Northeastern Brazil: Patterns of disease, nutritional impact, etiologies, and risk factors. Journal Infectious Diseases, 148: 986-997.

HABICHT, J. P.; MARTORELL, R.; MOLINA, R. M.; YARBROUGH, E. \& KLEIN, R. E., 1974. Height and weight standards for preschool children. Lancet, 1: 611-615.

HOYLE, B.; YUNUS, M. \& CHEN, L. C., 1980. Breast-feeding and food intake among children with acute diarrheal diseases. A merican Journal of Clinical Nutrition, 33: 2365-2371.

KAPLAN, E. L. \& MEIER, P., 1958. Nonparametric estimation from incomplete observation. Journal of the American Statistical A ssociation, 53: 457-481.

KLEINBAUM, D.; KUPPER, L. \& MORGENSTERN, H., 1986a. Measureof diseases frequency: Incidence. In: Epidemiologic Research. Principles and Quantitative Methods (D. Kleinbaum; L. Kupper \& H. Morgenstern, eds.), pp. 96-116, New York: Van Nostrand Reinhold Co.

1986b. Others measures of diaseases

frequency. In: Epidemiologic Research. Principles and Quantitative Methods (D. Kleinbaum; L. Kupper \& H. Morgenstern, eds.), pp. 117-139, New York : Van Nostrand Reinhold Co.

MARTORELL, R. \& HABICHT, J. P., 1986. Growth in early childhood in developing countries. In: HumanGrowth (F. Falkner \& J. M. Tanner, eds.) pp. 241-262, New York: Plenum.

MARTORELL, R.; YARBROUGH, C.; LECHTING, A.; HABITCH, J. P. \& KLEIN, R., 1975. Diarrheal diseases and growth retardation in preschool Guatemala children. A merican J ournal of Physical Anthropology, 43: 341-346.
MATA, L., 1983. Epidemiology of acute diarrhea in childhood. In: Acute diarrhea: its nutritional consequences in children (J. A. Bellanti, ed.), pp. 3-22, New York: Nestle Raveen Press.

MATA, L.; URRUTIA, J. J.; ALBERTAZZI, E.; PELLECER, O. \& ARELLANO, E., 1972. Influence of recurrent infections on nutrition and growth of children in Guatemala. A merican Journal of Clinical Nutrition, 25: 1267-1275.

OPS (Oragnización Panamericana de Salud), 1987. $M$ anual detratamiento de la diarrea. Washington, DC: OPS. (Serie Paltex, 13)

RIVERA, J. \& MARTORELL, R., 1988. Nutrition, infection and growth Part I: Effects of infection on growth. Clinical Nutrition, 7: 156-162.

RIVERA, J., 1988. Effect of Supplementary Feeding Upon the Recovery From Mild-to-M oderate Wasting in Children. $\mathrm{PhD}$ Thesis, Ithaca, New York: Division of Nutritional Sciences, Cornell University.

RIVERA, J.; MARTORELL, R. \& LUTTER, C. K., 1989. Interacción de la ingesta y la enfermedad diarreica en el crecimiento de los niños. In: B reastfeedind, Nutrition, Infection and Growth in Developed and Emerging Countries (S. A. Atkinson; L. A. Hanson \& R. K. Chandra, eds.), Canadá: Arts Biomedical Publisher and Distributors. (in press)

ROCHE, A. F.; GUO, S. \& MOORE, W., 1989. Weight and recumbent lenght from 1 to 12 months of age: Reference data for 1 month increments. A merican Journal of Clinical Nutrition, 49: 599-607.

ROWLAND, M. G. M.; COLE, T. J. \& WHITEHEAD, R. G., 1977. A quantitative study into the role of infection in determining nutritional status in Gambean village children. British Journal of Nutrition, 37: 441-450.

ROWLAND, M. G. M.; ROWLAND, S. G. J. G. \& COLE, T. J., 1988. Impact of infection on the growth of children from 0 to 2 years in an urban West African Community. A merican Journal of Clinical Nutrition, 47: 134-138.

WATERLOW, J. C.; ASHWORTH, A. \& GRIFFITHS, M., 1980. Faltering in infant growth in less develop countriees. Lancet, 2: 1176-1178.

WATKINSON, M., 1981. Delayed onset of weaning diarrhea associated with high breast milk intake. Transactions of the Royal Society of Tropical Medicine and Hygiene, 75: 432-435.

WHITEHEAD, R. G. \& PAUL, A. A., 1984. Growth charts and the assessment of infant feeding practices in the Western World and in developing countries. Early Human Development, 9: $187-207$ 